\title{
A modified Tessari method for producing more foam
}

Jie Xu ${ }^{1,2}$, Yi-fei Wang ${ }^{1}$, An-wei Chen ${ }^{3}$, Tao Wang ${ }^{3}$ and Shao-hua Liu $3,4^{*}$

\begin{abstract}
This study aimed to develop a modified Tessari method for producing more sclerosing foam in treatment of extensive venous malformations. Sclerosing foam was produced by using Tessari method and the modified Tessari method. The procedure of the later was as follows: prepared foam in a sclerosant-air ratio of 1:4; connected three disposable $10 \mathrm{ml}$ syringes to two medical three-way taps; drawn $4 \mathrm{ml}$ of liquid sclerosant into one syringe and $16 \mathrm{ml}$ averagely of air into the other two; then moved the plungers of all syringes back and forth for 20 times to produce sclerosing foam. The volume and foam half time (FHT) of foam produced by the two methods were compared. The average volume of sclerosing foam produced by Tessari method and the modified Tessari method were 9.8 and $19.7 \mathrm{ml}$, and assessed to have statistical difference. The FHT of foam produced by the two methods were 120 and $150 \mathrm{~s}$, and assessed to have statistical difference. In conclusion, the modified Tessari method could produce more fresh and stable sclerosing foam.
\end{abstract}

Keywords: Sclerosing foam, Tessari technique, Modified Tessari method, Sclerotherapy, Venous malformations

\section{Background}

During the treatment of venous malformations (VMs) using foam sclerotherapy, one of the most popular methods to produce foam was the Tessari method which used pumping cycles of liquid and air in-and-out of a double syringe system (Nastasa et al. 2015; Wolmann 2010; Tessari 2001, 2005). In clinical treatment, sclerosing foam was prepared usually by using two $10 \mathrm{ml}$ syringes; however, no more than $10 \mathrm{ml}$ of sclerosing foam could be produced at a time in this way. Tessari method was originally used in the treatment of varicose veins, in most cases of which $10 \mathrm{ml}$ of fresh sclerosing foam might be sufficient (Tessari et al. 2001). However, this volume might be insufficient in treating some VMs, especially when the region of lesion was extensive. Sufficient fresh sclerosing foam was needed for the treatment of extensive VMs.

There were several ways to get more fresh sclerosing foam, for example, producing foam once more, using bigger syringes or more operators and devices to produce simultaneously. However, all these methods had more or

\footnotetext{
*Correspondence: Ishabccba@126.com

${ }^{4}$ Department and Institute of Dental Medicine, Qilu Hospital, Shandong University, Wenhuaxi-Road, Jinan 250012, People's Republic of China Full list of author information is available at the end of the article
}

less defects. Here, we reported a modified Tessari method by using three $10 \mathrm{ml}$ syringes which could produce more fresh foam at a time; the volume and the stability of foam produced by the two methods were compared. Foam half time (FHT), the time when half of the original liquid sclerosant was reverted to liquid state, was adopted to assess the stability of the foam.

\section{Methods}

Disposable $10 \mathrm{ml}$ syringes, medical three-way taps and $1 \%$ lauromacrogol injection (Shanxi Tianyu Pharmaceutical Co., Ltd.) were used in this study.

Procedure of Tessari method (Tessari et al. 2001): drawn $2 \mathrm{ml}$ of liquid sclerosant into one $10 \mathrm{ml}$ syringe and $8 \mathrm{ml}$ of air into the other $10 \mathrm{ml}$ syringe; connected both syringes to a medical three-way tap; then, moved the plungers of both syringes back and forth for 20 times to produce sclerosing foam (Fig. 1).

Procedure of the modified Tessari method: drawn $4 \mathrm{ml}$ liquid sclerosant into a $10 \mathrm{ml}$ syringe and $16 \mathrm{ml}$ air averagely into the other two $10 \mathrm{ml}$ syringes; then connected all syringes to two parallel medical three-way taps; then moved the plungers of all syringes back and forth for 20 times to produce sclerosing foam (Fig. 2).

\section{Springer}

(c) 2016 Xu et al. This article is distributed under the terms of the Creative Commons Attribution 4.0 International License (http:// creativecommons.org/licenses/by/4.0/, which permits unrestricted use, distribution, and reproduction in any medium, provided you give appropriate credit to the original author(s) and the source, provide a link to the Creative Commons license, and indicate if changes were made. 


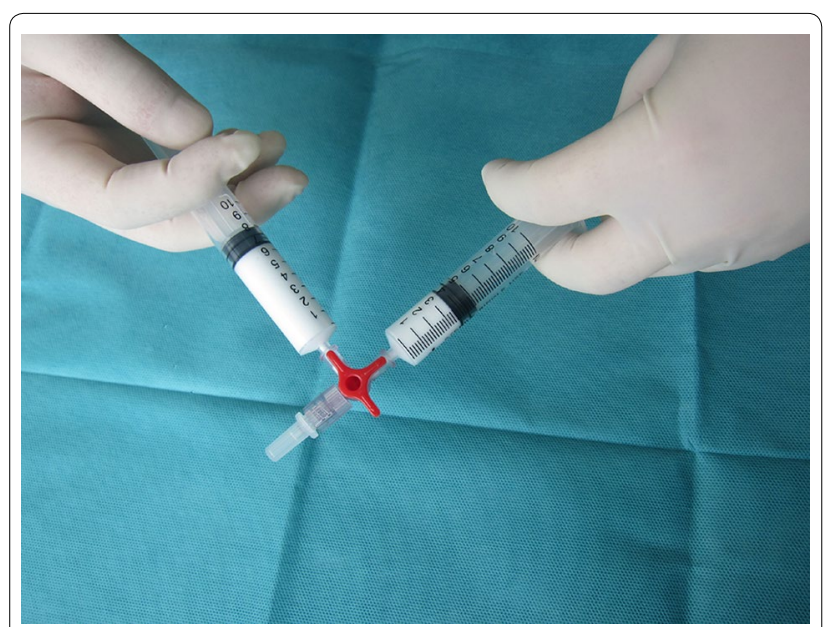

Fig. 1 Tessari method

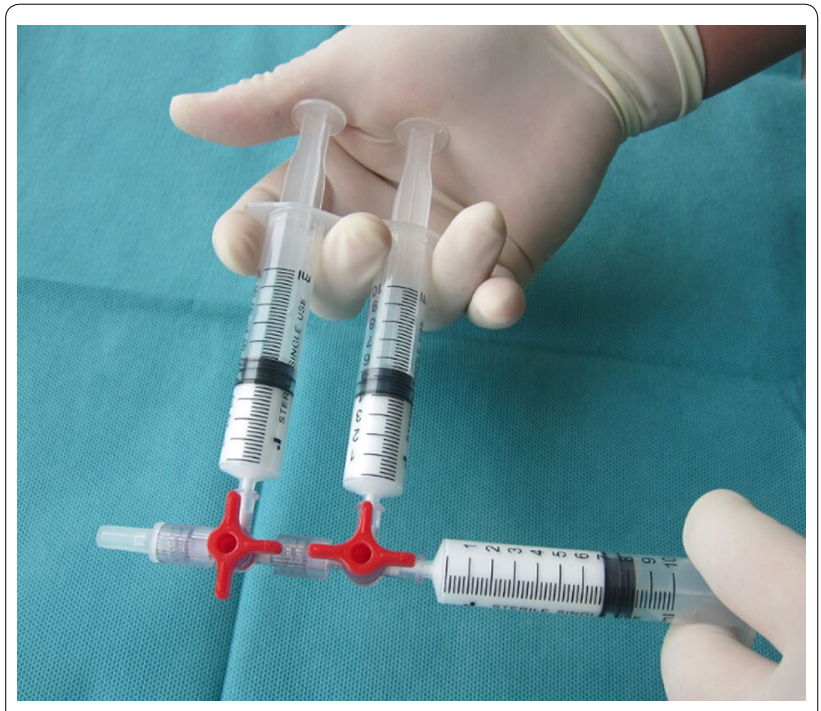

Fig. 2 The modified Tessari method

Detection of the foam volume and the foam FHT: Once the foam was produced, it was immediately pushed into 10 and $20 \mathrm{ml}$ scalar syringes respectively, the scalar syringes were placed vertically with the piston beneath. Macroscopic appearance of the foam was observed and volume of the foam was recorded. The foam in the syringes gradually separated into liquid and air, with the liquid sclerosant gathered at the bottom, when half volume of the liquid sclerosant was reverted, the time as FHT was recorded.

The detection above was measured respectively for 10 times by the same operator. Each foam was prepared by using new sclerosant and all experiments were performed at an ambient temperature of $25^{\circ} \mathrm{C}$. Experimental values were compared by an independent-samples $t$ test and analysed using SPSS 19.0. A $p$ value $\leq 0.05$ was considered significant.

\section{Results}

Right after production, the bubbles in foam were small and homogenous, when the foam began to re-convert to a liquid and air separated mix, foam bubbles got bigger and inhomogeneous, eventually foam converted to two components: liquid and air.

The average volume of sclerosing foam produced by Tessari method and the modified Tessari method were 9.8 and $19.7 \mathrm{ml}$, and assessed to have statistical difference.

The FHT of foam produced by Tessari method and the modified Tessari method were 120 and $150 \mathrm{~s}$, and assessed to have statistical difference (Table 1).

\section{Discussion}

Owing to its high efficiency, safety and economical advantages, foam sclerotherapy was widely used in treatment of vascular abnormalities (Luebke and Brunkwall 2008; Myers and Roberts 2009; Park et al. 2014; Van der Vleuten et al. 2014; Wolmann 2010). The high efficiency of foam sclerotherapy was closely related to the physical obstruction of blood flow and the displacing the intravascular blood, which extended extent of contaction between the sclerosants and the vessel wall (Ikponmwosa et al. 2010; Wolmann 2010; Eckmann 2009; Van Deurzen et al. 2011). Therefore, the therapeutic effects related to whether the cavity of VMs could be replete by fresh sclerosing foam; In our clinical work, sometime more than $10 \mathrm{ml}$ fresh sclerosing foam was needed for extensive lesion, some authors found that $10 \mathrm{ml}$ or more sclerosing foam was safety and efficacy, and no serious complications were observed (Stimpson et al. 2012; Chen et al. 2015). In our experience, the most common complications were temporal pain with injection, swelling after injection and skin ulcer in some patients.

In our experimental and clinical experience, it was inconvenient to use $20 \mathrm{ml}$ syringes to produce foam; and other authors had reported that $10 \mathrm{ml}$ syringes were the best choice to produce foam considering normal handling and foam stability (Wolmann 2010; Nastasa et al.

Table 1 Average volume and foam half time (FHT) of foam produced by Tessari method and modified Tessari method

\begin{tabular}{lcc}
\hline & $\begin{array}{l}\text { Tessari } \\
\text { method }\end{array}$ & $\begin{array}{l}\text { The modified } \\
\text { Tessari method }\end{array}$ \\
\hline The average volume $(\mathrm{ml})^{\mathrm{a}}$ & $9.8 \pm 0.2$ & $19.7 \pm 0.3$ \\
The FHT $(\mathrm{s})^{\mathrm{a}}$ & $120 \pm 4.13$ & $156 \pm 6.85$ \\
\hline
\end{tabular}

a The average volume and FHT were expressed as mean values \pm standard deviation 
2015). Tessari method using two $10 \mathrm{ml}$ syringes had become one of the most popular ways to produce foam. In clinical treatment, no more than $10 \mathrm{ml}$ of foam could be produced at a time by using two $10 \mathrm{ml}$ syringes, which might be insufficient in treating some extensive VMs.

To get more foam at a time, we might repeat the producing procedure once more or add more operators and devices. But the process of injecting foam into extensive VMs might be interrupted. During the interval between two adjacent injections, the previous foam would be washed away by the blood. Therefore, injecting sufficient foam at a time into the cavity of extensive VMs produced better therapeutic effects (Van Deurzen et al. 2011). Furthermore, there was another disadvantage when using the same syringe to prepare foam for the second time, it would be hard for the plunger to slide smoothly during preparation and injection. Adding more operators and devices to produce foam simultaneously meant to use more human and material resources. The modified Tessari method could produce about $20 \mathrm{ml}$ fresh foam at a time which, in our clinical experience, would be sufficient in treating some of extensive VMs.

FHT was a key and usually used parameter of the stability of sclerosing foam. In this study, the FHT of sclerosing foam produced by the modified Tessari method was longer than that by Tessari method, showing that the sclerosing foam produced by the modified Tessari method was more stable.

\section{Conclusions}

The modified Tessari method could produce more fresh and stable sclerosing foam at a time which was profitable in treating extensive VMs.

\section{Abbreviations}

VMs: venous malformations; FHT: foam half time.

\section{Authors' contributions}

All authors read and approved the final manuscript.

\footnotetext{
Author details

1 School of Stomatology, Shandong University, No. 44, Wenhuaxi-Road, Jinan 250012, People's Republic of China. ${ }^{2}$ Department of Oral and Maxillofacial Surgery, Stomatology Hospital of Jinan, No. 101, Jingliu-Road, Jinan 250012, People's Republic of China. ${ }^{3}$ Department of Oral and Maxillofacial Surgery and Institute of Dental Medicine, Qilu Hospital, Shandong University, No. 107, Wenhuaxi-Road, Jinan 250012, People's Republic of China. ${ }^{4}$ Department and Institute of Dental Medicine, Qilu Hospital, Shandong University, Wenhuaxi-Road, Jinan 250012, People's Republic of China.
}

\section{Acknowledgements}

This work was supported by the National Natural Science Foundation of China (No. 81172572), the Key Research and Development Project of Shandong Province (No. 2015GGH318012) and Basic Research of Qilu Hospital (2014QLKY).

\section{Competing interests}

The authors declare that they have no competing interests.

Received: 29 October 2015 Accepted: 12 February 2016

Published online: 19 February 2016

\section{References}

Chen AW, Liu YR, Li K, Wang T, Liu SH (2015) Efficacy of sclerotherapy with radio-opaque foam guided by digital subtraction angiography for the treatment of complex venous malformations of the head and neck. $\mathrm{Br} J$ Oral Maxillofac Surg 53(9):809-813

Eckmann DM (2009) Polidocanol for endovenous microfoam sclerosant therapy. Expert Opin Investig Drugs 18:1919-1927

Ikponmwosa A, Abbott C, Graham A, Homer-Vanniasinkam S, Gough MJ (2010) The impact of different concentrations of sodium tetradecyl sulphate and initial balloon denudation on endothelial cell loss and tunica media injury in a model of foam sclerotherapy. Eur J Vasc Endovasc Surg 39:366-371

Luebke T, Brunkwall J (2008) Systematic review and metaanalysis of endovenous radiofrequency obliteration, endovenous laser therapy, and foam sclerotherapy for primary varicosis. J Cardiovasc Surg (Torino) 49:213-233

Myers KA, Roberts S (2009) Evaluation of published reports of foam sclerotherapy: what do we know conclusively? Phlebology 24(6):275-280

Nastasa V, Samaras K, Ampatzidis Ch, Karapantsios TD, Trelles MA, MorenoMoraga J, Smarandache A, Pascu ML (2015) Properties of polidocanol foam in view of its use in sclerotherapy. Int J Pharm 478:588-596

Park Sang Woo, Yun Ik Jin, Hwang Jae Joon, Lee Song Am, Kim Jun Seok, Chee Hyun Keun, Chang II Soo (2014) Fluoroscopy-guided endovenous sclerotherapy using a microcatheter prior to endovenous laser ablation: comparison between liquid and foam sclerotherapy for varicose tributaries. Korean J Radiol 15(4):481-487

Stimpson P, Hewitt R, Barnacle A, Roebuck DJ, Hartley B (2012) Sodium tetradecyl sulphate sclerotherapy for treating venous malformations of the oral and pharyngeal regions in children. Int J Pediatr Otorhinolaryngol 76(4):569-573

Tessari L (2001) Extemporary sclerosing foam according to personal method: experimental clinical data and catheter usage. Int Angiol Suppl 1:54

Tessari L (2005) Nouvelle technique d'obtention de la scle'ro-mousse. Phlébologie 53:129

Tessari L, Cavezzi A, Frullini A (2001) Preliminary experience with a new sclerosing foam in the treatment of varicose veins. Dermatol Surg 27:58-60

Van der Vleuten CJ, Kater A, Wijnen MH, Schultze Kool LJ, Rovers MM (2014) Effectiveness of sclerotherapy, surgery, and laser therapy in patients with venous malformations: a systematic review. Cardiovasc Intervent Radiol 37:977-989

Van Deurzen B, Ceulen RP, Tellings SS, van der Geld C, Nijsten T (2011) Polidocanol concentration and time affect the properties of foam used for sclerotherapy. Dermatol Surg 37:1448-1455

Wolmann JC (2010) Sclerosant foams Stabilities, physical properties and rheological behavior. Phlebologie 39:208-217 